\title{
Research on the Key Technology of Distributed Network Management System
}

\author{
Xu Ran1, a, Ming Zhong1, b, Liang Qiao2, c, Fang Cheng1, d, Yali \\ Xiong3, e, Jia Liu1, f \\ 1 Network Information Center, Training Department, Chongqing \\ Communication College, Chongqing, 400035, China \\ 2College of Biomedical Engineering, Third Military Medical University, \\ Chongqing, 400035, China \\ 3Information Technique Management Center, Training Department, \\ Chongqing Communication College, Chongqing, 400035, China \\ aemail:361760350@qq.com, bemail:rfox@sina.com, cemail: \\ bobqiao@163.com, \\ demail:cf-cq@163.com,eemail:459224626@qq.com, \\ femail:307477266@qq.com
}

\begin{abstract}
With the increasing of large-scale network and complex structure, the traditional network management modes and means fail to meet the needs of large-scale network management, the distributed network management system is in urgent need to assist managers to complete the daily network management and maintenance, this paper presented a mode of campus-network distributed network management system , and made in-depth discussion and analysis on such key technologies as data communication, topology discovery and failure management of the distributed network management system.
\end{abstract}

Keywords: Network Management; Distributed Network Management System; Failure Management

\section{Introduction}

With the rapid development of information technology, the campus network is characterized by increasing application systems and network services, larger scale, more complicated system structure and increasing heterogeneous structure. In order to achieve the goals of network management, network managers must make clear about the real-time current operation of the network, find the security threats and the failures in the network as early as possible. Through intelligent analysis and diagnosis , they must know and locate the network failure, send out timely warning, and take rapid response measures[1].Therefore, the traditional network management modes and means cannot meet the needs of large-scale network management, and the distributed network management system is in urgent need to assist managers in completing the daily network management and 
maintenance, so as to improve the quality and efficiency of the network operation and provide better services for the users.

\section{Distributed network management system mode}

In order to meet the requirements for scalable network and hierarchical network management, the distributed network management system mode adopts multilevel network architecture. The functions of the whole system are completed by the five parts: the management center, management workstation, Agent, MIB and network management protocol together to.

Within the campus network, a management center is set up to manage all the network equipment of the campus, Meanwhile, the management center provides a variety of user interface, enabling the managers to get a vivid image of the entire network operation.

In terms of the physical structure of the campus network and virtual local area network, the network equipment is subdivided into several management areas. In each area, a management workstation is set up to monitor and control the operation of its own network equipment, storage and process the collected monitored data and then submit them to the management center while accepting the coordinated scheduling by the management center and assisting the later in the network management.

Agents involve in the whole process of the network equipment management, monitoring and controlling the resident devices. Agents can interact with the management workstations, constantly monitor and respond to query from the management workstation or set commands. If agents find errors and other abnormalities, they report the errors to the management workstation directly rather than wait for polling of the later .

MIB is a collection of objects that provide the data on the managed network devices. Each object is generally a data variable, representing a certain aspect information of the managed network devices. The information on the network equipment acquired by agents are all derived from the MIB[2].

The network management protocol SNMP is used for packaging commands and messages between the workstations and agents, standardizing the rules and data format of information interaction between them and simplifying the complicated process of the network management.

The main characteristics of distributed network management system mode are as follows: the whole network adopts a management center; many management workstations playing dual roles; the management workstations display distributed layout, the nearest deployment, and the entire network conducts coordination through the management center. The management area of each management workstation is a part of the whole network management. This effectively reduces the workload of the management center, avoid the collapse of the system resulting from the single point failure, and achieve the purpose of the distributed management[3]. The mode of distributed network management system is as shown in Figure 1. 


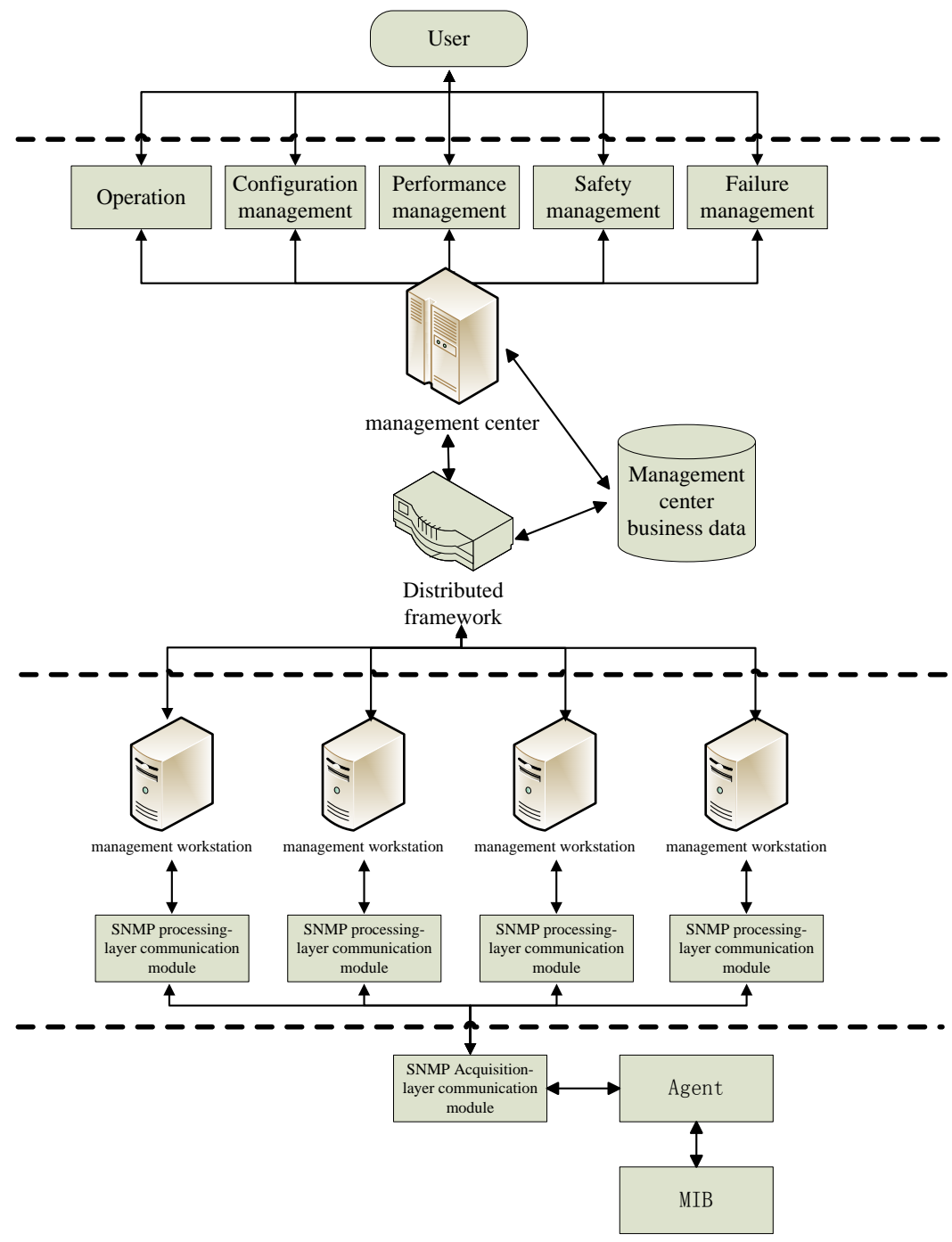

Figure 1 Mode of distributed network management system

\section{Analysis and discussion about the key technologies of the distributed network management system}

With the distributed network management system, a large number of network devices turn into active management from passive acceptation of management. This management mode has greatly overcome the disadvantages of the traditional centralized network management system, able to meet the management requirements for flexible expansion of the large-scale network and improving the flexibility and reliability of the network management system. 
However, in the process of real-time network management, the key problems urgent to be solved are data communication, topology discovery, failure management among the hierarchical systems.

(1) Studies on data communication

In the mode of the distributed network management system, the management center is mainly liable for management of the whole network, including configuration management, performance management, safety management and failure management. All the network devices within the network are subdivided into several management areas. Each area is controlled by a management workstation. The management center gets information on the operation of the network devices from management workstations, makes management commands, collaborates with all the management workstations to work together .Each management workstation monitors and controls the operation of its own network equipment, storages and processes the collected monitored data and then submits them to the management center while accepting the coordinated scheduling by the management center and assisting the later in completing the tasks of the network management.

The management center can assign management tasks to the management workstations by sending out such instructions as Get immediate, Set immediate, Get scheduled, and Set Scheduled while the management station must make realtime monitoring on the instructions sent out by the management center, ready to complete the management task assigned.

The management workstation makes dynamic polling on the devices under control, acquires the data on the operation of the devices by adopting the following five operations in the SNMP protocol: get-request, get-next-request, set-request and get-response. Besides, the center analyzes, filters and sorts out the data and finally storage them in the local database of the management station. It feedbacks the processed results to the management center in accordance with the management instructions from the management center .

For the acquired important data, such as equipment anomaly, fault or failure, the management workstation will take the initiative to report alarming information on the devices to the management center, inform them the network failure and assist the network managers in time troubleshooting.

(2) Research on topology discovery

The distributed network management system covers a large number of management workstations and network devices which can make dynamic access to the network and can be adjusted upon request. Therefore, the distributed network management system must have the function of topology discover, automatically discovering the newly-accessed network devices and send the data on the new devices to all the nodes of network management, enabling all other nodes of management can get the data on the new devices including routing, naming and organization. Meanwhile, the distributed network management system needs to make a real-time management on the establishment, withdrawal, movement and reconstruction of the network nodes, coordinate the network communication and information transmission between devices, ensuring the safety and reliability of data transmission. 
The distributed network management system can complete the work of topology discovery with the SNMP protocol messages, and the management center sets the parameters and periods of topology discovery and assigns tasks to the management workstations which implement topology discovery periodically in accordance with the requirements of the tasks. The management center, the management workstations and the controlled devices use MIB information to store the data on their own devices. All the data communication between the devices within the network is exchanged through the MIB messages. Once the management station detects the changes of the network nodes, it should immediately feedback the parameters about the changes of the network nodes to the management center. The center will timely the update network topological structure according to the received information on the devices and, therefore completing the task of topology discovery .

(3) Research on failure management

In the distributed network management system, the network devices in every area are under the control of their own independent management workstation. Any failure of the management workstation may make a large number of network devices within the local area out of control. In order to improve the reliability and availability of the distributed management system, the failure management of the workstation is a urgent and difficult problem for the distributed network management system to solve.

After the distributed network management system is established, each management workstation obtains the table for the addresses of the global network devices by exchanging data with the management center and with other management workstations .All the management workstations make periodically queries about the working situations of each other. Once a management workstation fails due to failure, another management workstation which has detected the failure will report it to the management center and applies to be a temporary manager of the failed area. If the management center receives report requests from more than one management workstations, it will, by comparing the received message timestamps, select the workstation with the earliest failure timestamp to control the work of the failed workstation as an agent while rejecting the applications from other workstations. In this way, the distributed network management system can be redeployed and complete the network management.

\section{Performance analysis of distributed network management system}

We use MPT to quantitative analysis of the performance of the distributed network management system, MPT can be defined as a network event generation to be processed the time[4], it can be expressed as

$$
\begin{aligned}
& \mathrm{MPT}=(1 / \mathrm{P}) /(1-\mathrm{g} *|\sqrt[\mathrm{L}]{\mathrm{N}}| / \mathrm{P})+\sum_{i=2}^{L}\left(\left(1 / \mathrm{P}_{i}\right) /(1-\right. \\
& \left.\left.\left(G_{i} * R_{i} *|\sqrt[\mathrm{L}]{\mathrm{N}}|\right) / \mathrm{P}_{i}\right)\right) \\
& \mathrm{N} \text { is the number of network devices management, g is each network device }
\end{aligned}
$$


that generated the event frequency, L is the level of Distributed network management system, $\mathrm{P}$ is the processing ability of each management workstation, $\mathrm{R}$ is the probability of each network event to the upper transmission, and $\mathrm{R} \leq 1$ 。

when $\mathrm{i}>2, \mathrm{G}_{\mathrm{i}}=\mathrm{G}_{\mathrm{i}-1} * \mathrm{R}_{\mathrm{i}-1} *|\sqrt[\mathrm{L}]{\mathrm{N}}| ; \mathrm{G}_{2}=\mathrm{g} * \mathrm{R}_{1} *|\sqrt[\mathrm{L}]{\mathrm{N}}| ;$ when $\mathrm{L}=1$, System degradation for centralized network management system, and when $\mathrm{L}=2$, The upgrade of the system is a distributed network management system. In order to analyze the performance indexes of two kinds of network management system, We set the parameters $\mathrm{P}=1000, \mathrm{~g}=10, \mathrm{R}=0.5$. The performance of the centralized network management system and distributed network management system comparison as shown in Table 1, with MPT milliseconds.

Table 1 Performance comparison of centralized network management system

\begin{tabular}{|c|c|c|c|c|c|c|c|c|c|c|c|c|}
\multicolumn{10}{c|}{ and the distributed network management system } \\
\hline \multirow{2}{*}{ Centrali } & $\mathrm{N}$ & 1 & 10 & 20 & 30 & 40 & 50 & 60 & 70 & 80 & 90 & 100 \\
\cline { 2 - 15 } zed & $\mathrm{MP}$ & 1.0 & 1.1 & 1.2 & 1.4 & 1.6 & 2.0 & 2.5 & 3.3 & 5.0 & 10. & 102 \\
& $\mathrm{~T}$ & 1 & 1 & 5 & 3 & 7 & 1 & 2 & 5 & 2 & 1 & \\
\hline \multirow{3}{*}{ Distribu } & $\mathrm{N}$ & 1 & 20 & 60 & 10 & 50 & 100 & 200 & 500 & 800 & 900 & 100 \\
ted & & & & & 0 & 0 & 0 & 0 & 0 & 0 & 0 & 00 \\
\cline { 2 - 13 } & $\mathrm{MP}$ & 2.0 & 2.0 & 2.1 & 2.1 & 2.5 & 2.6 & 3.1 & 5.0 & 10. & 22. & 101 \\
& $\mathrm{~T}$ & 5 & 8 & 3 & 9 & 1 & 8 & 2 & 1 & 98 & 50 & \\
\hline
\end{tabular}

As can be seen from Table 1, The centralized network management systems can only be used for small-scale network management, and the distributed network management system greatly expands the number of network devices management, furthermore the distributed network management system can also be further expansion of network management scale by increasing the management level[5].

\section{Conclusion}

This paper presented a mode of the distributed network management system based on SNMP, and the key technologies of the distributed network management system were analyzed and discussed. The system was realized on the.NET platform with C\# language.

A long time testing operation proves that the system framework is flexible, scalable and stable, effectively improving the efficiency of network management and fully meeting the needs of large-scale network management.

\section{Acknowledgement}

This study was supported by the Natural Science Foundation Project of CQ CSTC(NO:cstc2011jjA10032).

\section{References}

[1] Hong F, Bai Y. Performance evaluation of wireless mesh networks with selfsimilar traffic[A]. Wicom 2007, The 3rd IEEE International Conference on 
Wireless Communications, Networking and Mobile Computing[C]. Shanghai, China, 2007.1697-1700.

[2] Chris Hobbs, A Practical Approach to WBEM/CIM Management, Auerbach Publications, 2012.

[3] He CL, Dang XC. A distributed network fault management model based on SNMP. Computer Programming Skills \& Maintenance[J].2011(10):115-116.

[4] Wang P, Zhao H, Li L. A distributed network management oriented adaptive scalable model. Journal of China Institute of Communications[J].2002, 23(12):118-128.

[5] WU Z M, DU S. Customer Premises Network and Community Broadband Integrated Services[M]. Beijing: Posts and Telecom Press,2007. 